\title{
ESCRIBIR EN VENECIA EN EL SIGLO XVIII
}

\author{
WRITING IN VENICE IN THE 18TH CENTURY
}

Teresa Gil García

Universidad Complutense de Madrid

\section{Resumen:}

El pensamiento ilustrado que define la estructura social de Venecia en el siglo XVIII, favorece la participación de la mujer en la vida ciudadana. A este espacio público al que accede desde sus exigencias naturales de educación, reconocimiento y libertad, consigue llegar porque es capaz de expresar cuanto considera digno de ser contado y transmitido para que la sociedad misma, de receptora de esfuerzos y logros, se convierta en la deudora de tantos méritos suyos. La osadía intelectual de algunos nombres históricos, demuestra que talento y esfuerzo sirven para que la vida sea también expresión de voluntad propia. $Y$ con todo, se reivindica el derecho a ser feliz, que no es poco, como felices han sido las respuestas artísticas -teatrales y musicales- de otros participantes en estos debates fervientes sobre la condición femenina. Otra cosa hubiera sido que todo hubiera quedado en premisas teóricas, deliciosas para una discusión, pero poco útiles en la construcción de un mundo nuevo.

\section{Palabras claves:}

Venecia, siglo XVIII, escritoras, teatro, música.

\section{Abstract:}

The Enlightenment that defines the social structure in Venice in the 18th century advocates women's participation in public life. Women get to accessing this public sphere, from their own natural needs of education, recognition and freedom, because they are able to express what they consider worthy of being told and passed on so that society itself, as the beneficiary of efforts and achievements, eventually turns into the debtor of their numerous merits. The intellectual boldness that pertains to some historically renowned thinkers and writers shows that talent and effort are aimed at making life the very expression of one's own will. That also implies the vindication of the right to happiness, in the same way as the different artistic responses by those who participated in such fervent debates over women's condition -in both the fields of music and theatre- have been happy. If this had been confined merely to theoretical assumptions -undoubtedly interesting for some discussion, yet not so useful towards the construction of a new world- would have been a different matter.

\section{KEY WORD:}

Venice, 18th century, women writers, theatre, music. 
Chi no sa viver a Venezia, no sa viver in nissuna parte del mondo (Carlo Goldoni, La buona moglie, acto I, escena VIII)

\section{LA CIUDAD}

En la Venecia del siglo XVIII, por mor del destino, aparentemente casi todo iba mal. Imposible era esconder su decadencia en barrios de familias venidas a menos -los barnaboti-y ridículo se percibía además conservar los antiguos privilegios estamentales a quienes la divina providencia había regalado la exención del trabajo. Si había sido poderosa y rica, ahora era minúscula ante los vecinos amenazadores, pues política y económicamente no podía ya hacer frente a sus obligaciones. El Settecento veneciano se perfilaba pues como un tiempo de nostalgia por edades doradas, por un mundo que se deshacía en un proceso imparable que imaginaba, en cambio, otro mejor.

Y sin embargo, es un momento dulce de su historia, pues la ciudad se había apartado de batallas irresolutas y de combates enquistados, y había asumido otros compromisos menos exigentes y más rentables. Se había vuelto una tierra escogida para convivir:

\section{Sotto sto benedetto cielo i sgherri e i malviventi no i trova protezion, e certe bula e, che se usa lontan de qua, a Venezia no le se pratica, e no le se pol praticar", diría uno de los tantos Brighella de las comedias de Goldoni, e incluso una meta de viaje perfecta para llevar a buen término hasta una utopía: "chi no sa viver a Venezia, no sa viver in nissuna parte del mondo (Goldoni: La buona moglie, acto I, escena VIII).}

A las bondades del lugar contribuye claramente una clase social emprendedora e industriosa que ya había dado muestras de afrontar el trabajo como algo digno y necesario para mantener equilibradas las arcas ciudadanas. Alguna vez ya lo había conseguido a través de los caminos inescrutables de la edición de textos y la imprenta; también la ayudaron las transacciones mercantiles, que no estuvieron reñidas en absoluto con aquella devoción comunicativa primigenia, pues sobre una base veneciana se había formado una lengua franca en el Mediterráneo oriental; y las más de las veces, la tolerancia de las tierras bajas de la Laguna había aceptado sin rechistar la contribución económica de ingresos provenientes del juego o de placeres mundanos. Profundo era el pasado, pero no un misterio. Esta fuerza emprendedora, con una base muy creativa, se asentaba en un tejido social en el que prosperidad equivalía a esperanza. Y esperanza, no memoria, anuncio, espera, promesa, es la primera categoría de la conciencia histórica, como argumentaba Hegel por entonces. Así pues, con toda la confianza depositada en la suerte y en la estabilidad ciudadana a la que contribuía tranquilamente, sin todavía percibir el papel que iba a desempeñar en el futuro, una nueva clase social de burgueses irrumpe en la historia, pues hasta entonces había estado marginada por oscuros designios del compromiso cívico, a pesar de que sus miembros eran muchos y bien organizados. Y tentando a los diablos y su engañoso
Antiguo Régimen, aquellos industriosos venecianos ponen en el cuadro de análisis la situación, de modo que su estilo de vida, la lengua que aprecian y usan, la ilusión que el arte les ofrece, se conviertan en valores que defender. Sin temor a perder su causa, ni la confianza y el aprecio que se habían ganado con tanto esfuerzo, ellos mismos procurarán que las huellas del pasado excelso de las tradiciones más arraigadas de su ciudad se conviertan en el molde de un presente mejorado, a pesar de que las palabras decadencia y declive merodean alrededor.

Ser veneciano resultaba ser una suerte, lo decía Goethe. Los habitantes de esta ciudad habían gustado de la escritura y de los juegos, los placeres, la insolencia y la gloria; y no se contentarían con menos. Tenían un compromiso al que debían responder en lo que en sus manos estaba y así lo hicieron, con las palabras, con la música, con la pintura, que son precisamente aquellas manifestaciones donde el ser humano es verdaderamente libre, por responsable. Sentimiento y pasión, como valores intrínsecos del ser humano, son los pilares que debían mantener esta civilización. A esto se añade que el pensamiento ilustrado del siglo XVIII establecía nuevos fundamentos para la convivencia. Además, en la situación que se perfilaba, el nuevo espacio social representado por esta clase emergente de burgueses exige una distribución de compromisos en los que, al menos en teoría y a su modo, todos participen, también ese cincuenta por ciento de la población que solía quedar al margen, que son las mujeres. Y como estamos en el siglo XVIII, la razón y lo razonable sitúan a la mitad de esta ciudadanía en un justo medio que modifica las estructuras sociales y familiares.

\section{VOCES FEMENINAS}

Aunque la presencia femenina es indicio saludable de equilibrio social, la sociedad burguesa y su distribución de roles, todavía limitaba su existencia en su proyección personal a un espacio privado, sin posibilidad de acceder a la esfera pública, a no ser de la mano de su marido. El eje fundamental de la sociedad burguesa veneciana gravitaba en torno a este planteamiento. No obstante, estas perspectivas de cambio introdujeron un aire distinto en la vida de las mujeres, y poco tiempo después ya sería indiscutible su incorporación a la educación y al trabajo, gracias al esfuerzo y la tenacidad de aquellas que abrieron el camino a las demás, al haber demostrado ser merecedoras de respeto y reconocimiento. En Venecia existía ya una tradición de mujeres comprometidas con su tiempo, hecho que constituye una excepción en Italia, incluso en Europa. Sin caer en un excesivo protofeminismo, hay que reconocer, no obstante, que todas ellas pertenecían a una misma clase social privilegiada y poseían una capacidad de expresión, de participación en la vida cultural y de hacer llegar sus escritos a la imprenta que no eran comunes (Bellavitis 2008). 
Por lo demás, en Italia, durante todo el siglo, se había generado un amplio debate sobre la cuestión femenina, y en Venecia, incluso, con mayor virulencia porque el ambiente era propicio para las polémicas, como si la limitación de los canales cercara a los intelectuales y su escape natural fuera a través de palabras encendidas: "Quel che colpisce a Venezia è la varietà e la veemenza delle opinioni, come se la stretta coabitazione nel centro lagunare attizzasse fra i letterati il gusto della replica e della contraddizione" (Fido 1984: 45); y porque aquí había materia sobre la que seguir discutiendo. Las mujeres venecianas habían estado siempre muy presentes en la vida social de la República, como demuestran todo tipo de documentos de la actividad práctica, que nos ofrecen pistas para construir modelos ejemplares. Ni era desconocida en la ciudad una tradición de escrituras en femenino, ni la dedicación a otras artes, a la pintura o a la música. En esta promoción de la educación es ejemplar la existencia de orfanatos de niñas, a las que, como actividad educativa para poder llevar de adultas una vida digna, se les enseñaba a tocar un instrumento, a cantar, o simplemente a transcribir notas sobre papel pautado. El mismo Vivaldi, profesor en el Ospedale della Pietà, compuso para esta institución algunas piezas. La historia nunca las olvidó, y recientemente la novela de Tiziano Scarpa (2009), Stabat Mater, nos ha traído a la memoria las posibilidades que ofrece el arte para que las cosas cambien, al principio imperceptiblemente y luego ya con fuerza irresistible. Si detrás de las rejas del orfanato, todo ocurría en sigilo, más visible era, en cambio, un grupo conspicuo de mujeres cultas que había sido capaz de establecer una red social de manifestaciones literarias, con matices políticos a veces, en salones y academias, y se había introducido en el mundo del periodismo y de la edición, aprovechando de todas las oportunidades de intervenir en estas altas esferas culturales (Lampron 2008). Otro dato que ayuda a entender por dónde iban discurriendo los caminos de la presencia femenina es la importancia de Venecia en la cultura europea. La ciudad se había convertido en meta privilegiada del Grand Tour y la región va a acoger intelectuales venidos de toda Europa que también contribuirán a resaltar la vocación cosmopolita de la Laguna. Este privilegio no pasaba desapercibido tampoco a ilustres ciudadanos que querían destacar todo lo que de beneficioso e interesante ofrecían estas actividades a la convivencia.

De buenas y múltiples maneras y con más o menos acierto nos han llegado los textos de quienes evitaron el olvido de la meritoria condición femenina. Estos escritos, que se pueden vincular a una tradición de escrituras feministas avant la lettre y que circulaban por toda Italia desde la Edad Media, representan un filón de pensamiento que supone una valoración de la naturaleza y de la dignidad femeninas y su defensa por parte de prudentes intercesores de su condición, como el mismo Torquato Tasso, autor del famoso tratado Della virtú femminile e donnesca (1582). Estos textos se solían redactar con dos objetivos: como compensación al descuido o a la escasa estima de los méritos femeninos, o, en su versión mejorada, para resaltar la contribución de los personajes reseñados a la convivencia -y a la cultura- general. En este espíritu encomiástico entendemos, por ejemplo, el epitalamio de Bartolommeo Gamba escrito para la boda de su amigo Iacopo Crescini, donde hace una excelente defensa de las virtudes de la mujer véneta, ejemplificadas en las ilustres de la época: “Voi pregherò a voler con grazioso sorriso accogliere le brevi Vite che danno anima e forma a questo libretto. Vi ricorderanno esse il merito di alcune illustri donne delle nostre vineziane contrade, delle quali vedete ad un tempo delineate anche le varie fattezze" (Gamba 1826). Entre las mencionadas, nombra a una notable veneciana, Luisa Bergalli, a quien reconoce el mérito de ser la editora de una "raccolta delle più illustri rimatrici d'ogni secolo, con cui fe' conoscere che può essere dato alle donne, sì bene che agli uomini, di altamente cantare". Evitando el tono moralista dominante en el género biográfico del siglo XIX, Gamba recordaría años después de su muerte a aquella mujer que había sido responsable de su vida, manejando afanes, ganancias y sinsabores sin valimiento, que es como resume el biógrafo las vicisitudes vitales de la esposa de Gasparo Gozzi. Pero el reconocimiento de los méritos y valores femeninos no estuvo ausente de polémicas. Efectivamente las mujeres podían reclamar su espacio, o una habitación propia, aunque las resistencias eran muchas y de mucha envergadura todavía. Sin embargo, en el siglo XVIII, las luces de la razón ofrecen un camino despejado a las discusiones pues garantizan viveza de espíritu y el ingenio y la dialéctica necesarios para defender las posiciones y convocar a interlocutores cada vez más interesados y estimulados por los argumentos que se aducen. La suerte es que los debates tenían además una gran proyección en los medios intelectuales y en los foros culturales: en el teatro y en las tertulias donde la oralidad permite el encuentro simultáneo de las voces participantes, y en los textos escritos de varia naturaleza, en la prensa periódica o en la misma literatura, que acoge los temas palpitantes aunque con otro carácter. Y así poco a poco, se perfilan nuevas formas de libertad, apoyadas en un esfuerzo de escrituras femeninas, que son claros indicios de modernidad.

La polémica sobre la condición femenina era un debate abierto que seguramente apasionaba, como lo son los temas candentes de actualidad en que se enfrentan dos grupos bien definidos y en igualdad de condiciones, digamos. Todas las preguntas que se puedan hacer sobre la orientación y el cariz que las exigencias de los nuevos tiempos demandaban en relación con la educación femenina y sus consecuencias, van a encontrar respuestas razonables. Contra la opinión tradicional sobre la condición de la mujer que la limitaba a sus tareas consabidas y la recluía en casa, sin participar plenamente en la vida social, se impone la realidad. La osadía intelectual de las mujeres afortunadamente va unida al asentamiento del ideario ilustrado y al progreso correspondiente, que acabaría finalmente por ser factor definitorio de la nueva estructura social. 
Las opiniones del matrimonio Gozzi, Luisa y Gasparo, son paradigmáticas del debate que provocaba la presencia cada vez más visible de voluntariosas mujeres en la vida pública. Sus argumentos, si no enfrentados, sí responden de manera distinta a la cuestión. El marido, Gasparo, que parece no atreverse a entrar en materia, matizaba su opinión sobre la diferencia de los sexos y de su comportamiento aduciendo las evidentes razones de la naturaleza: “[essa] non potea metter nell'uomo forza virile e delicata belleza ed ella compose un uomo forte e una donna bella... ella fa un uomo che studia e una donna che danza con leggiadria" (G. Gozzi, 1967: 91): el estudio es asunto fuerte y masculino y la danza, delicada y femenina, porque así era la condición de ambos. El razonamiento de Gozzi no era original, pues se hacía eco de las teorías de un experto en materia que había tenido un gran calado en la ideología ilustrada, Jean Jacques Rousseau. Este pedagogo suizo había señalado diferencias razonables en las pautas educativas de los jóvenes protagonistas de su ensayo Emile ou de l'Éducation. En el libro V, a Sofía, la ficticia enamorada del Emilio, le reserva la tarea de completar su formación a través de la institución del matrimonio siguiendo siempre los consejos y designios del marido. El ideal educativo del filósofo ginebrino confirmaba la dependencia de la mujer y su limitación al ámbito familiar, pues es a esta institución a la que debe pertenecer por naturaleza y razón, es decir las vidas femeninas deben orientarse hacia la esfera doméstica y privada (Rousseau 1762).

Luisa defendía, en cambio, el derecho a elegir vida y vocación de acuerdo con la indiscutible igualdad intelectual. Tampoco era extraña esta exigencia porque en Venecia las mujeres gozaban ya de una libertad desconocida en otros lugares. Y no sólo las de condición más favorecida, sino otras de posición más modesta sabían acceder a su promoción por otros cauces, en sus limitadísimas parcelas domésticas. La fuerza de los argumentos de la Bergalli para defender sus designios reside en su propia voluntad de escribir, empeño que la libera de todas las ataduras impuestas, pues el principio motriz de la escritura es precisamente el deseo de salir de las filas de lo oscuro, como bien explicaría Kafka mucho después. Con estas buenas intenciones, a modo de clara justificación de su bien argumentada teoría, y para que no se perdieran en el paso del tiempo poemas salidos de las plumas femeninas, prepara Luisa una antología que ha puesto un nombre que recordar, y en un lugar en la historia de la literatura, a predecesoras en sus mismos afanes literarios: Componimenti poetici delle più illustri rimatrici di ogni secolo (1726). La recopilación de estas composiciones es la consecuencia del esfuerzo personal de la Bergalli por demostrar que las mujeres cuando tienen a disposición los medios necesarios, son capaces de lograr una meta con facilidad. La mejor defensa de su tarea se apoya, pues, en esta selección de poetisas y poemas que considera deben estar presentes en una manifestación de brillantes resultados de inspiración y trabajo. Conmemorar a las predecesoras en su tarea de escritoras se traduce en un florilegio de mujeres virtuosas capaces de servir de modelo a generaciones futuras, reclamando a la vez una identidad veneciana propia, que encaja muy bien en las fechas en que se redactaba. Aquí podemos encontrar la explicación de por qué convenía leer estos textos, y qué lecciones se podían extraer de la lectura. Esta antología se abre con una composición de Moderata Fonte (Bergalli 1726) pseudónimo acuñado como expresión transparente de quien aspira a ser manantial, fuente de vida y conocimiento, que esconde a Modesta Pozzo. Escribir bajo pseudónimo no era propio de mujeres, ya que rara vez ingresaban en las academias y por tanto no podían asumir esta identidad bucólica. Pero en este caso, nuestra poetisa en simulada apariencia, se atreve a glosar en sus Tredici canti di Floridoro, a la manera de Ariosto, las supuestas limitaciones de la condición femenina, especialmente aquellas que podían mitigarse por la educación. Y no es baladí el razonamiento: Si la naturaleza ofrece generosamente sus dones a hombres y mujeres sin distinción, ¿por qué habrían de ser ellas diferentes en sentimiento y razón?

Le Donne in ogni età fur da Natura

Di gran giudicio, e d' animo dotate,

Nè men atte a mostrar con studio, e cura

Senno, e valor degli Uomini son nate.

E perchè, se comune è la figura,

E perchè, se comune è la figura,
Se non son le sostanze variate,

Se non son le sostanze variate,
$S^{\prime}$ hanno simile un cibo, e un parlar, denno

Differente aver poi l' ardire, e il senno?

(Bergalli 1726)

Y si en su naturaleza, idénticos eran hombres y mujeres, ¿por qué no habrían de dedicarse ellas también con la misma pasión a actividades intelectuales? La cuestión estaba clara. El mejor ejemplo de que Moderata Fonte tenía razón era precisamente esta muestra de escrituras que seguía los versos de introducción: no es raro que una mujer escriba y que lo haga bien. Luisa Bergalli Gozzi, Irminda Partenide en su nombre arcadémico, fue tan adelantada en su época que sufrió incluso una vocación frustrada la de no poder escribir cuanto, como, donde y cuando quería, como ha subrayado su biógrafa (Élisabeth Ravoux-Rallo 2001: 159-166). Y a pesar de ello, ocupa su espacio en la literatura, entre otras cosas, por esta tarea suya tan acertada de organizar una antología de poetisas en lengua italiana; de esta manera también ella misma se reconocía como parte del grupo de mujeres activas en un ambiente cerrado, capaces de historia literaria. El trabajo lo tiene que distribuir en dos tomos, pues lo minucioso de la reseña y la mención de más de doscientas cincuenta escritoras le obligan a la atenta distribución del contenido. El primer tomo alcanza hasta el año 1575 y el segundo está dividido a su vez en dos secciones, una continúa el eje cronológico y la última incluye los textos de las contemporáneas colocadas en orden alfabético. La antología se la dedica al cardenal Pietro Ottoboni, miembro de la Accademia de la Arcadia y mecenas de artistas, poetas y músicos, con el sencillo deseo de que la lectura de estos textos sirvieran a evitar al menos el prejuicio de que "in noi Donne regnar non possa 
talento, onde nelle bell' arti distinguersi, e segnalarsi". En realidad, la benevolencia que espera Luisa del cardenal tiene un mayor significado, pues implica el reconocimiento igualitario de la pluma masculina o femenina, en el centro mismo del poder artístico, que era la corte vaticana, y no se caracterizaba precisamente por la defensa de la causa de las mujeres.

Aunque no es difícil imaginarse la formación de un escritor, en este caso la reconstrucción de las vicisitudes literarias de mujer como Luisa Bergalli nos lleva a pensar en una joven que tendría gran fuerza de voluntad y carácter para seguir un camino duro y conseguir su meta. Su nombre sonaba más en la ciudad que el de Gasparo, aun después de casados, pues parece ser que se le reconocía en Venecia como el marido de la Bergalli. Lo cierto es que también había tenido buenos maestros como Apostolo Zeno, a quien seguiría en su otra vocación de autora de tragedias (Martín Clavijo 2011), y Antonio Sforza, al que había ayudado en la edición de sus poemas. Y su obra también ha quedado ligada, cómo no, a la de su marido Gasparo (Crotti 1989).

El trabajo lo había llevado a cabo Luisa, con paciencia, imaginamos, y con dos puntos exiguos de referencia, que ella anota en la advertencia a los lectores, las antologías de Domenichi y Giovan Batista Recanati, el Teleste Ciparissiano de la Accademia della Arcadia. Su contribución por eso es palmaria. La antología pasa revista a quinientos años de historia literaria, inevitablemente a partir del siglo XIII, recorriendo escuelas y géneros y ofreciendo datos biográficos de las autoras. Como toda selección subjetiva, a parte de recoger los nombres de todas las escritoras cuyo nombre conserva el paso del tiempo, incluye otras composiciones anónimas, con lo que la antología se organiza en torno a criterios que la misma autora explica. El suyo es un trabajo presidido por el gusto, por placer personal de trabajo cumplido, "perché mi lusingo di acquistare a me ancora un qualche compatimento", y porque escoge las composiciones que más parece apreciar de aquellas poetisas que ella, y la historia después, no tienen intención de olvidar. A pesar de que no hay criterio más parcial que el placer, por subjetivo; sin embargo, Luisa Bergalli prepara esta antología desde una motivación trasparente, y esto es la sagrada tarea y el compromiso de todo autor con sus lectores. En aquellos tiempos de cambio, escribir es, y lo matiza en un poema encomástico dedicado a las tres hermanas Carriere, una tarea elevada que exige esfuerzos, pero libera a la mujer de otras condenas:

Voi, che spregiate il gentil sesso, voi

D' Angela di Rosalba, e di Giovanna

Venite a mirar l' opre, e dite poi.

Dite pur s' io mentisco; e se $\mathrm{m}^{\prime}$ inganna

La passione, e dite pur se noi

Donne all' ago, ed al fuso il Ciel condann

(Bergalli 1726)

Condenada estaría Luisa, en cambio, al lugar de las mujeres nefastas si de ella sólo hubiéramos sabido lo que su cuñado Carlo Gozzi escribió en las primeras páginas de sus Memorias inútiles, por no se sabe qué motivos. La pinta como una mujer de imaginación desbordante, ambiciosa y desmesurada en sus aspiraciones e incapaz de lograr un equilibro entre su actividad pública y sus obligaciones domésticas. El caso es que aun así, anotando lo desastrosa que parecía ser su vida privada, el segundo de los Gozzi liga sin querer su recuerdo al suyo con las ataduras de una pizca de envidia que no ha pasado desapercibida a cuantos leemos estas líneas:

Questa femmina di fervida e volante immaginazione, e perciò, abilissima a'poetici rapimenti, volle per i stimoli d'un buon animo, misti con quelli dell'ambizione e della presunzione che aveva della sua attività, inoltrarsi a regolare le cose domestiche disordinate; ma i suoi progetti e gli ordini suoi non poterono uscire da ratti romanzeschi e pindarici Innamoratasi d'un dominio ideale e divenuta sovrana d'un regno tisico, col desiderio di far tutti felici, con verace disinteresse, altro non d'un regno tisico, col desiderio di far tutti felici, con verace disinteresse, altro non fece che tessere delle maggiori infelicità a tutti gli altri, non meno che a se medesima
(Gozzi, Carlo 1797-98).

\section{Las RAZONES Masculina}

La denostación de los méritos femeninos y el rechazo a admitirlas en actividades que por tradición no ejercían, no es excepcional en el sigloXVIII. Entre los ilustrados italianos había un grupo de misóginos -podríamos llamarlos así-, abierta o subrepticiamente declarados en contra del derecho a que ellas escogieran libremente un camino artístico e intelectual (Giordano 1994: 5). Afortunadamente los misóginos son personajes poco conocidos, lo que demuestra también cuán trasnochadas estaban sus opiniones. Pero de entre estos había incluso quien se atrevía a dar un paso al frente. Por ejemplo, el profesor de Filosofía Antonio Volpi se declaró contrario al ingreso de la mujer en el estudio de las Ciencia y de las Bellas Artes durante la querelle ocurrida en la Accademia dei Ricovrati en Padova en 1723. La respuesta a su tesis la defendió Aretafila Savini de' Rossi, escritora de Siena, con su Apologia in favore degli Studj delle Donne (Trapani 2010: 1-11).

Pero otra representación, más acorde con el momento histórico, se mostraba a favor de la educación femenina y lo que pudiera conllevar. Afortunadamente estos eran los más, y sobre todo aquellos que mejor manejaban los resortes de la pluma para transmitir opinión: Giuseppe Baretti, que defendía honorablemente actitudes difícilmente comprensibles a los extranjeros que visitaban Italia (Baretti 1769); Cesare Beccaria, Saverio Bettinelli, Giuseppe Parini; Antonio Piazza, autor de una novela, Il teatro ovvero Fatti di una veneziana donde cuenta su experiencia como hombre de teatro en el ambiente vivo de la Venecia del siglo (1778); Pietro Chiari o el mismo Carlo Goldoni. La opinión de la que podían participar los venecianos se formaba, entre otros cauces, a través del teatro, que como espacio de diversión y tranquilidad, podía servir sencillamente a interesar al público sobre sus circunstancias vitales. El teatro es el 
espacio consagrado a transmitir la imagen de la mujer moderna. Es el punto en que concuerdan vida e imaginación, instante capaz de dar forma a la progresiva afirmación femenina en la estructura familiar y en la organización social. Esta correspondencia entre mundo y teatro es la base sobre la que construir el entramado educativo e ideológico de las obras, que deben, a su vez, agradar a los espectadores -y cuantos más mejor- a través de los recursos propios del espectáculo. Si así lo entiende el autor teatral, habrá conseguido su éxito. Venecia mejor que ningún otro sitio acertaba en la transmisión de estas experiencias, porque aquí se daban las condiciones para que este experimento triunfara, público acostumbrado a las representaciones, autores atentos a su mundo y dispuestos a preparar textos para todos, temas generados con el fermento ideológico del momento y tiempos adecuados a tales encuentros. Y como fin primordial de los responsables, agradar a los espectadores, pues por su naturaleza espectacular, el teatro responde también a la lógica aspiración a la felicidad del hombre y del ciudadano, un principio de la Enciclopedia que afecta a todos por igual, hombres y mujeres, y que merodeaba en el ambiente.

Así que, como instrumento indicado para acercar el público al mundo, el teatro podía exponer el estado de la cuestión de la educación y someter a juicio los comportamientos que se percibían. De este modo, se hace eco de las inquietudes femeninas por escribir. Y por leer, porque en equilibrio estable con la escritura, la lectura es la otra cara del esfuerzo, con la suerte de que las lectoras, como las espectadoras, son una categoría más numerosa. De este modo, esta incipiente combinación de mujer y cultura llega a hacerse visible, lo que ayuda efectivamente a la causa. Con mucho esmero, los autores cuidan de su público. Goldoni en sus Memorias defendía que convenía empezar por atraer a las mujeres a los espectáculos: "je faisois ma cour aux dames de mon pays, mais j'agissois pour mon intérêt en même temps, car pour plaire au public, il faut comencer par flatter les dames" (Mémoires, v. II: 119), actitud que es una prueba más de un cambio de mentalidad y que acrecienta la aceptación -pasión incluso- de estas manifestaciones. Antonio Piazza o el abate Piero Chiari se mostraron también hábiles constructores de situaciones ingeniosas que tuvieron una cierta acogida, el segundo más que el primero, entre el público femenino acostumbrado ya a verse protagonista de muchas vicisitudes. Goldoni, que se ganaba el favor y el reconocimiento popular con el estudio minucioso de las mujeres en escena, ya anciano confesaría que por conveniencia, como hemos visto, también tuvo su especial aportación al debate ideológico que canalizaba la opinión de los venecianos, afrontando con mesura la polémica. Hubiera sido anacrónico oponerse al reconocimiento del derecho de las mujeres a la educación a la libertad de sus designios, a estudiar, a escribir. Lo que planteaba el autor es cómo ponderar sus méritos, cómo valorar su relación con el mundo y el papel que ella debe resolver en la familia, que son como hemos visto los dos ejes sobre los que se construye la mentalidad burguesa. Y buscar luego el equilibrio entre los dos extremos, el lugar que a la mujer le había reservado la tradición y las oportunidades que la cultura del siglo le ofrecía. La novedad era la educación, por la que acceder a un estatus superio y al reconocimiento de sus méritos, como condición indispensable para su promoción. Así que la educación y el objetivo de esta educación es la cuestión que se resuelve en los dramas de Goldoni: el autor traduce los efectos del cambio de mentalidad en una sociedad, que debe hacer cuentas todavía con el pasado.

El comediógrafo veneciano, que es un inmejorable pintor de caracteres, es el mejor artífice de la imagen de sus contemporáneas. Crea mujeres resueltas, voluntariosas, dueñas de su propio destino, que trazan y defienden con inteligencia y sensatez. Esta perspectiva artística les ofrece una esperanza vital muy interesante. Precisamente, la primera comedia escrita enteramente a la que aplica su reforma teatral es La donna di garbo, donde una Rosaura con sensibilidad e ingenio, y la ayuda de su buena educación verá el triunfo de sus razonamientos sobre el sexo masculino y los prejuicios del pasado. Conviene Goldoni en que lo ha hecho así porque:

Chi è quegli che abbia coraggio di affermare non darsi delle Femmine dotte e virtuose? Lo smentirebbero tutte quelle sagge ed erudite Signore, che si a dotte e anc à anche a' ditalia dove io d'Italia dove io sono stato, e finalmente in tútta il carattere di una Femmina la quale, benché dotta, pure è soggetta a tutte le umane passioni (Goldoni: La donna di garbo, L'autore a chi legge).

Y sin embargo el argumento principal que defiende el autor, y que retoma en muchas otras comedias como en La buona moglie, continuación de La putta onorata; La moglie saggia, La bella giorgiana o incluso en La locandiera donde el tema que se debate es la libertad de la mujer para seguir su propia vida, eso sí, dentro de un orden, la educación no es asumida como valor absoluto, puesto que en la mujer está puesta al servicio de su felicidad, que en términos de la ideología burguesa de la época, solo puede encontrar en la esfera de lo privado. Goldoni no entra en las polémicas de la época y se mueve en una cierta ambigüedad. En un sentido de desinteresada generosidad con la excelencia femenina, elogia el talento de la mujer que se ha cultivado con esfuerzo y paciencia en el estudio, como hace con una amiga, María Gaetana Agnesi, famosa matemática de la época, profesora de la Universidad de Bologna, que le había dedicado una copia de su tratado matemático, Instituzioni analitiche ad uso della gioventù italiana, probablemente porque sabía de su interés por la formación de los jóvenes. Y no solo le da las gracias literariamente:

Stupitevi piuttosto, che con saper profondo

Prodotto abbia una donna un sì gran libro al mondo.

E italiana l'autrice, signor, non è olandese,

Donna illustre, sapiente, che onora il suo paese:

Ma se trovansi altrove scarsi i seguaci suoi,

Ammirasi il gran libro, e studiasi da noi (Il medico olandese, acto I, escena II). 
Más aún, a la amiga estudiosa le ofrece el tributo universal de la fama en esta escena segunda del primer acto de Il medico olandese, donde informa al público sobre la fortuna de Instituzioni en el extranjero, en Holanda, país en el que la obra era muy apreciada. Pero no siempre es así el comediógrafo veneciano, pues con otra actitud más común, más de acuerdo con el Mundo inspirador de su teatro, como él mismo resalta en sus Memorias, Goldoni las más de las veces se pliega al sentir de la mayoría y a la ideología dominante, y construye una mujer virtuosa, que somete a prueba su educación para el fin que ha sido llamada, que es el matrimonio (Rodríguez 2002: 339-350). Pero mientras tanto, el Iluminismo había aportado también unos efectos que se han hecho sentir: la mujer es cada vez más visible en sociedad y más partícipe de la gestión de la cultura, porque escriben, traducen, adaptan obras de teatro; redactan crónicas en los periódicos; se interesan por las ciencias experimentales, esperando el momento en que puedan estar plenamente comprometidas en la vida de todos.

\section{Conclusión}

El siglo XVIII es un momento interesante en la historia de la literatura italiana. Y Venecia es un lugar muy vivo, donde la mujer puede demostrar la fuerza que posee para construir su propio destino, para ser educada, y para hacer uso de la libertad que la época ofrece. Si además es capaz de expresar su opinión, de escribir y de contar lo que pasa, la sociedad pasará a ser incluso deudora. En estas manifestaciones de su personalidad y de su opinión se van a poder apreciar mejor talento y esfuerzo, que finalmente podrán brillar en las distintas artes que cultivan. La época está bien definida en sus principios. El empeño femenino por salir de los oscuro, daba sus frutos, y así lo demuestra el espectáculo popular del teatro, donde se reconoce y aplaude el derecho de la mujer a su educación y a hacer de su vida expresión de la voluntad propia. Lo bueno de ello es que además se reivindica ser feliz, que no es poco, como felices han sido las opiniones de los participantes en estos asuntos, pues han conseguido que tengan una expresión artística que podemos valorar ahora. Otra cosa habría sido si todo hubiera quedado en premisas teóricas, deliciosas para una discusión, pero poco útiles para que la sociedad que todavía hay que seguir construyendo.

\section{REFERENCIAS BIBLIOGRÁFICAS}

Baretti, G., An Account of the Manners and Customs of Italy, With Observations on the Mistakes of Some Travellers With Regard to That Country. 2 vols. London, 1769.

Bellavitis, A. Del Torre, G., et alii, "Donne a Venezia. Spazi di libertà e forme di potere (sec. XVI-XVIII)" - Atti del Convegno, Venezia, 8-10 maggio 2008. Ed. elec http://www.storiadivenezia.net/sito/index.php?option=com content\&view=article\&id=103:donne\&catid=39:sagg
Bergalli Gozzi, L., Componimenti poetici delle più illustri rimatrici di ogni secolo, Venezia, Ed. Eidos di Mirano, 2006 (1726). Ed. electrónica de la Universidad de Chicago: http://artflx.uchicago.edu/cgi-bin/philologic/getobject.pl?c.7:1:3.iww

Crotti, I., Ricorda, R., “Gasparo Gozzi. Il lavoro di un intellettuale nel Settecento veneziano", Atti del convegno, Venezia-Pordenone, 4-6 dicembre 1986, Padova, Antenore, 1989.

Fido, F., Da Venezia all'Europa : prospettive sull'ultimo Goldoni, Roma, Bulzoni, 1984.

Gamba, B., Alcuni ritratti di donne illustri delle province veneziane pubblicati in occasione delle Nozze di Iacopo Crescini padovano, con Adelaide Meneghini veneziana nel dì 15 gennajo, 1826 Edición electrónica en http://books.google.it

Giordano, A., “La letteratura femminile fra moda, mestiere e cultura”, en A. Giordano, Letterate toscane del Settecento. Un regesto, Firenze, All'insegna del Giglio, 1994.

Goldoni, C., Mémoires, ed. di Guido Mazzoni, 1907. También en books.google.es

----, Commedie, en ed. elect.: http://www.liberliber.it/libri/g/goldoni/index.htm

Gozzi, C., Memorie inutili, ed. de Domenico Bulfereti, UTET, Torino, 1923. Ed. elect.: http://www.unive.it/nqcontent.cfm?a_id=33861

----, Scritti scelti, ed. De Nicola, UTET, Torino, 1967.

Lampron, E. M., “Sociabilité et résautage entre les femmes de lettres vénitiennes, lagune et terre ferme (1770-1830) en Donne a Venezia, cit. Ed. elect. http://www.storiadivenezia.net/sito/donne/Lampron_Sociabilit.pdf

Martín Clavijo, M., "Luisa Bergalli: la querella de las mujeres y el teatro", La querella de las mujeres en Europa e Hispanoamérica, Sevilla, ArCiBel ed., 2011.

Piazza, A., Il teatro ovvero Fatti di una veneziana, edizioni Costantin, Venezia, 1778. Recientemente ha sido publicado bajo el título de La attrice en edición de Roberta Turchi, Napoli, Guida 1984

Ravoux-Rallo, E., Las mujeres en Venecia en el siglo XVIII, Madrid, Editorial Complutense, 2001.

Rodríguez Gómez, I., "Representación del mundo femenino en el universo teatral de Carlo Goldoni", Cuadernos de Filología, Anejo I, 2002.

Rousseau, J.J., Emile ou l'éducation, 1762. Ed. electrónica de la universidad de Columbia, texto francés e inglés: http://www.ilt.columbia.edu/pedagogies/rousseau/Contents2.html

Tasso, T., Della virtú femminile e donnesca, Sellerio, Palermo, 1997 (1582). En ed. electrónica: http://www.liberliber.it/mediateca/libri/t/tasso/discorso_della_virtu_etc/pdf/discor_p.pdf Scarpa, T., Stabat Mater, Torino,Einaudi, 2009

Trapani, E., “Profilo biografico di un' improvvisatrice toscana del Settecento: Fortunata Sulgher Fantastici", en Archivio di Stato, Firenze, en ed. electrónica: http://www.archiviodistato. firenze.it/memoriadonne/cartedidonne/cdd_14_trapani.pdf 